\title{
Contribution to Simplified Residual Stress Calculations of Multi-Layer Welds
}

\author{
Jakob Klassen ${ }^{1, a,{ }^{*}}$, Thomas Nitschke-Pagel ${ }^{1, b}$ and Klaus Dilger ${ }^{1, \mathrm{c}}$ \\ ${ }^{1}$ Langer Kamp 8, 38106 Braunschweig, Germany \\ aj.klassen@tu-braunschweig.de, bt.pagel@tu-braunschweig.de, ck.dilger@tu-braunschweig.de
}

Keywords: Residual Stresses, Welding Simulation, X-Ray Diffraction, Neutron Diffraction

\begin{abstract}
Especially for larger structures destructive measuring techniques for residual stress determination can be neglected and non-destructive methods are subject to disadvantages such as the limited resolution. Furthermore, residual stress gradients in the direction of sheet thickness cannot be determined easily.

The growing use of FEM, on the other hand, gives an insight into residual stresses at each point of the computed model. However, FEM for residual stress calculation is subject to certain limits and error sources due to the fine discretization and the high degree of non-linearity. In particular, the calculation of multi-layer welds will reach high computation times. This known issue is often counteracted with radical simplification of the numerical model such as lumping or semitransient method. The result inaccuracies of these methods are rarely quantified and published. To clarify which simplification strategies are applicable in numerical welding simulation reference models were produced and experimentally verified. On this basis, simplification approaches were investigated numerically and their effects on result quality were quantified.

It could be shown that most of the commonly used simplification approaches for the calculation of residual stresses and distortions are only partially permissible, if any. Each method has its limits and poses a risk to the user, if certain data for validation and verification are available only to a limited extent. This means that a strongly localized comparison between experiment and calculations is not necessarily a proof of correctness of the calculation approaches if a more refined experimental determination is dispensed with.
\end{abstract}

\section{Introduction}

Residual stresses are often suspected of having an influence on the service life of welded constructions, as those stresses are associated with crack formation and crack growth. In general, it is assumed that residual stresses superimpose with load stresses and thus can have a positive or negative effect depending on sign and value. Numerous works on these issues were published in the past. In any case, knowledge of the exact residual stress state is of great interest for the design of welded constructions. These exact stress states, however, are highly dependent on a multiplicity of factors. Therefore, a statement about the type and size of the respective residual stress distributions is not trivial. Thus, a compilation of representative residual stress distributions, for example in cataloged form, is desirable but not available for mentioned reasons.

There are numerous measuring techniques to obtain residual stresses. However, most techniques (e.g. diffraction methods, hole drilling etc.) are either expensive or limited in terms of accessibility, especially for through-thickness residual stress determination of thicker plates with possible residual stress gradients. Therefore, a reliable statement about the residual stress state in or near a critical location is often denied. These unavoidable experimental limitations may be overcome using numerical methods, in particular the finite element method (FEM). The FEM itself can be referred to as state of the art in calculation of residual stresses and distortion. 
Calculated results allow an insight in any desirable area of the computed model. However, volumetric models of multi-layer welds and larger structures result in large models and still unreasonable calculation times. Calculation engineers facing these problems are tempted to answer with a supposedly simple approach, namely simplifying dramatically. Common simplification approaches such as reduction of discretization, lumping or application of semitransient approaches do lead to manageable computation times. However, results thus obtained and therefore their benefits are highly doubtful.

In the scope of the present work several (most common) simplification approaches were applied on a reference case. This reference was validated and verified through abundant experimental investigations by means of temperature and distortion measurements as well as residual stress determination using $\mathrm{X}$-ray and neutron diffraction techniques.

Experimental investigations. Specimens' dimensions with a milled V-notch and welding parameters are given in Figure 1. For validation of numerical models temperature measurements were conducted. Type K thermocouples were used. Furthermore, distortions were obtained by means of laser trigonometry.

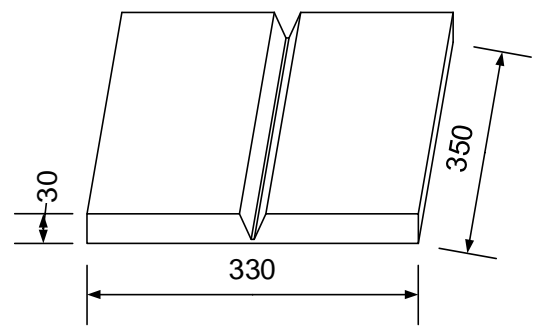

Welding parameters

$240 \mathrm{~A}$

$16,3 \mathrm{~V}$

$1,08[\mathrm{~m} / \mathrm{min}]$ wire feed

$10[\mathrm{~cm} / \mathrm{min}]$ welding speed

Figure 1: Specimen dimensions [mm] and welding parameters

Material properties. Experiments were carried out on a fine grained construction steel S355N with conspecific filler wire. This steel grade is commonly used in highly loaded constructions, e.g. steel bridges. Chemical composition and mechanical properties are given in Table 1.

Table 1: Chemical composition (by wt. [\%]) and mechanical properties of used steel grade

\begin{tabular}{|c|c|c|c|c|c|c|c|c|c|}
\hline & $\mathrm{C}$ & $\mathrm{Si}$ & $\mathrm{Mn}$ & $\mathrm{P}$ & $\mathrm{S}$ & $\mathrm{Cr}$ & $\mathrm{Ni}$ & Mo & $\mathrm{Cu}$ \\
\hline S355N: & 0,186 & 0,411 & 1,128 & 0,012 & 0,001 & 0,042 & 0,369 & 0,005 & 0,163 \\
\hline Tensile test: & \multicolumn{9}{|c|}{$\mathrm{R}_{\mathrm{eH}}=\sim 380 \mathrm{MPa} ; \quad \mathrm{R}_{\mathrm{m}}=\sim 540 \mathrm{MPa}$} \\
\hline
\end{tabular}

Residual stress measurement. Residual stresses (RS) are defined in reference to the welding direction. Thus, longitudinal RS are in welding direction and transverse RS perpendicular to the weld (Figure 2).

Near surface residual stresses were measured by means of X-ray diffraction (XRD). Diffraction lines ( $\{211\}$-patterns) were obtained with $\mathrm{CrK}_{\alpha}$-radiation using a $2 \mathrm{~mm}$ collimator. Afterwards residual stresses were calculated with the $\sin ^{2} \psi$-method according to [1], [2]. Through thickness residual stress measurements by means of neutron diffraction (ND) were carried out at the E3 instrument at Helmholtz-Zentrum Berlin. 


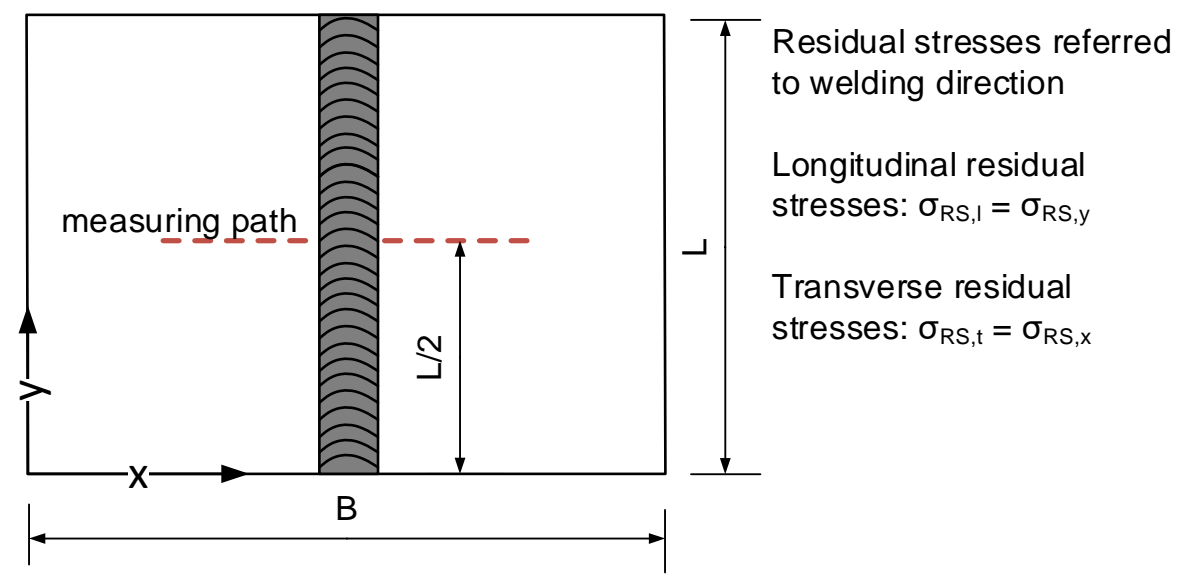

Figure 2: Orientation of measured and calculated residual stresses

Numerical investigations. Numerical welding simulations by means of three-dimensional fully transient structural finite element analyses were carried out with the SYSWELD code (Sysweld 2015.1, Version 17). Phase transformation was taken into account. Numerical models were calibrated by measured temperature fields and macrosections of each weld layer.

No clamping was applied, neither experimentally nor numerically. Therefore, deformations were inevitable. However, in numerical simulations the entire weld had to be modelled in advance ("chewing gum" or "quiet elements method" instead of "element birth method" - see [3], [4] and [5] for comparison) anticipating highly distorted elements of the final passes (according to [4]). Thus, elements' aspect ratio changes from bottom to the top layers, as shown in Figure 3. Corresponding mesh size information is given in Table 2. In further figures each FE mesh has been suppressed for clarity reasons.

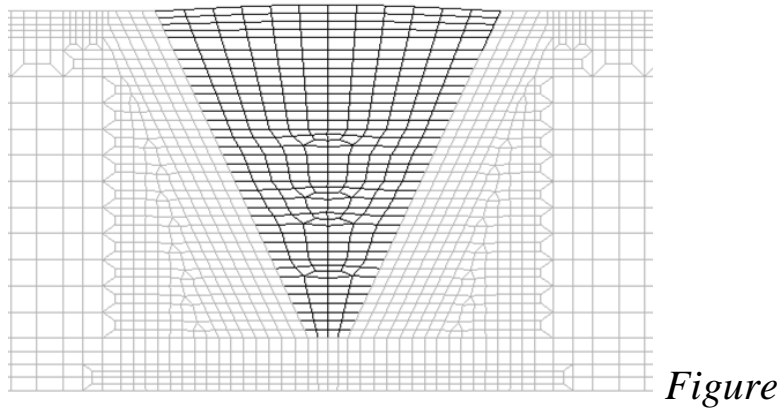

3: Finite element mesh cross section
Table 2: Details of FE mesh, V-model

\begin{tabular}{|l|l|}
\hline \multicolumn{2}{|l|}{ Reference model-V } \\
\hline \# of hex elements & 215308 \\
\hline \# of nodes & 224609 \\
\hline element size (x) [mm] & $0,75-2,2$ \\
\hline element size (y) [mm] & 3,0 \\
\hline element size (z) [mm] & $0,5-0,7$ \\
\hline
\end{tabular}

Simplification approaches. Some of most commonly applied simplification approaches are simplified geometries, applied thermal cycles as well as lumping several layers of multi-layer welds. However, there is hardly any scientific justification for some of these approaches. Within the framework of this paper these simplifications were implemented and compared with experimentally validated fully transient simulations. It is important to note that overall heat input in each model remained unchanged. This is a mandatory boundary condition, since calculation engineers only can rely on information obtained from welders or work shops.

Applied thermal cycle approach (TC). Weld build-up was adopted from V-model. Thermal cycles were experimentally obtained at certain points and assigned to numerical model. Thus, all elements of each pass were subject to certain thermal cycles (following measured values) at the same time. 
Lumping approach (L). FE meshes were adopted from V-model. In VL-model lumping was implemented such that all passes of each layer were summarized to one layer (compare Figure 4).

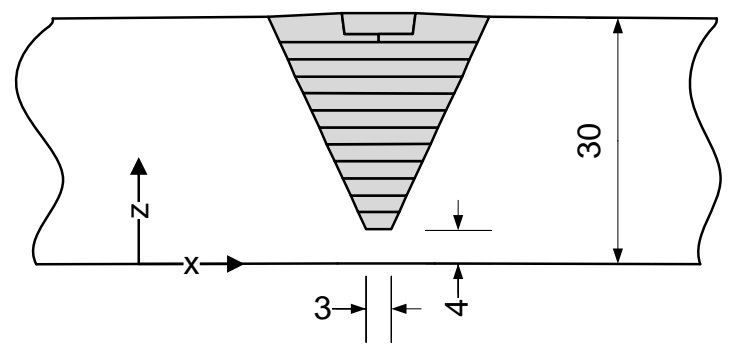

Figure 4: Weld build-up, VL-model [mm]

\section{Results}

The initial model (V) has been validated and verified with experimental data from temperature, distortion and residual stress measurements (comp. Figure 5). Thus, validation of the numerical model as well as its suitability for variations of further calculations is given (see [6] for further information).

It is important to note that points of residual stress measurements were linked by lines for clarity reasons only. Error bars are not plotted for the same reasons. However, errors' standard deviation of each measurement was calculated and was found to be less than $\pm 10 \mathrm{MPa}$ in average.
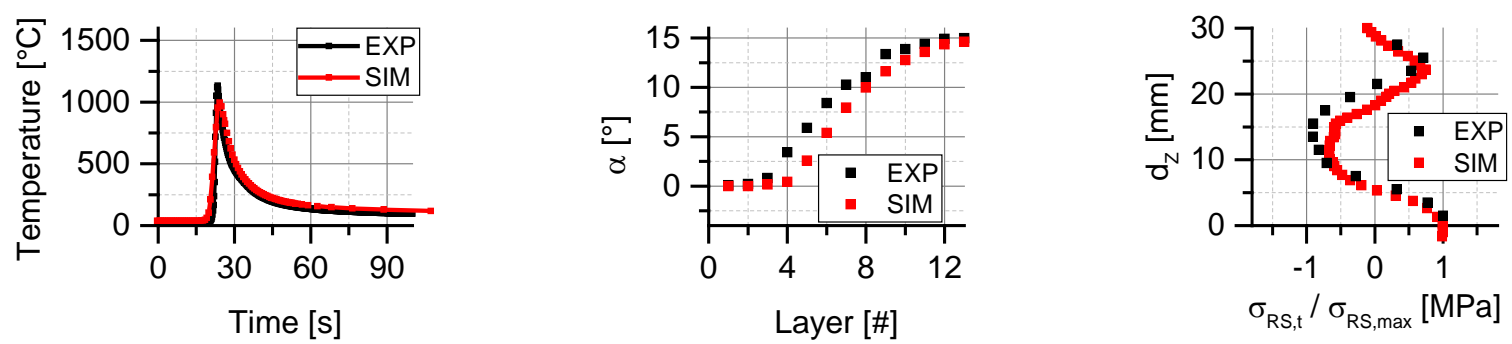

Figure 5: Examples for validation and verification of reference model-V

Applied thermal cycle approach. The results of a fully transient residual stress calculation with moving heat sources (model V) are compared with the results using the TC method (model TC). On the one hand, calculated longitudinal residual stresses at the bottom (not shown here) do not show significant differences. This was to be expected, since shrinking is equally hindered after first layer being welded. On the other hand, transverse residual stresses in the TC model are underestimated with an increasing number of layers. This effect is most likely to be explained with different conditions for hindered shrinkage in longitudinal direction when applying thermal cycle on the entire layer to be welded. Contrary to this, hindered shrinkage changes transiently with moving heat sources due to sequentially activation of filler wire elements. These influences of multi-layer thermal cycle approach on transverse residual stresses are even higher, as can be seen in Figure 6. Overall, transverse residual stresses are significantly smaller in magnitude and show even a change in sign.

Through-thickness residual stress distributions in the weld center are given in Figure 7. The assumptions stated above, in combination with tempering effects due to weld build-up, result in minor tensile residual stresses. Furthermore, the absence of plastic strains in transverse direction, which are mainly responsible for angular distortions, necessarily results in falsely predicted distortions. 


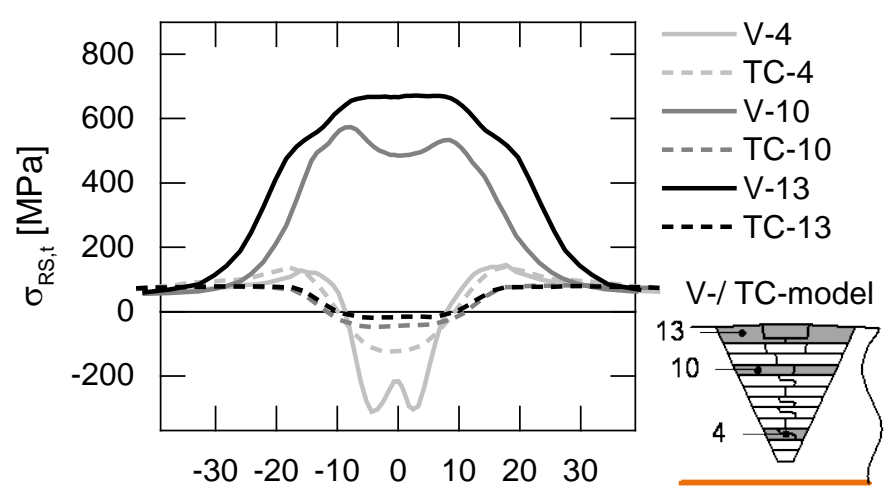

Distance from weld centre line [mm]

Figure 6: Comparison of calculated residual stresses (models $V$ and TC)

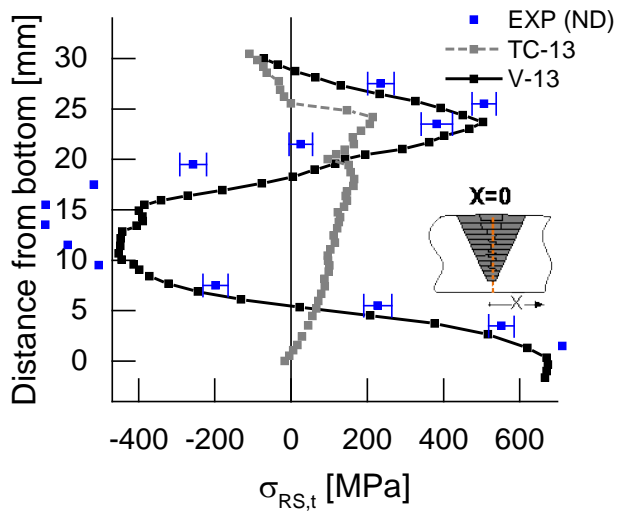

Figure 7: Comparison of calculated through-thickness residual stresses (models V and TC)

It can be noted that residual stress states of the transient calculation (which had been validated by measurements) is not reproduced in the TC approach, at all.

Lumping method. Lumping method was investigated on numerical basis only, for experimental validation is not possible. Calculated near surface residual stresses of one lumping approach were fairly comparable qualitatively. Distributions on each top side were comparable, which is due to equally modelled final passes. However, residual stresses in through-thickness direction show strong deviation in sign and value (see also Figure 8). Reasons for this are seen in increasing volumes of summarized passes which most likely are responsible for deeper penetrating annealing effects. Furthermore, these effects reduced plastic strains decreasing distortion.

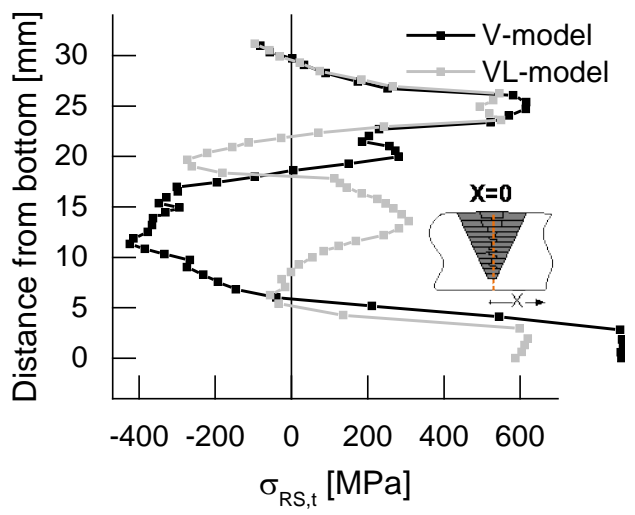

Figure 8: Comparison of calculated through-thickness residual stresses (models V and VL1)

It is important to note that calculated near surface residual stresses do not necessarily give information about the through-thickness distribution. Thus, obtained results might be misleading as was already found in a previous project [7].

\section{Conclusions}

In this paper simplification approaches have been presented that often can be found in literature. However, almost none of these publications do even try to validate their models. Instead, cherry picked experimental results are used for verification. Within the framework of this paper it could be shown that the investigated simplification approaches show less potential and more limitation 
in terms of result quality. For instance, it is well known that application-oriented simulation approaches need to keep energy input balanced. Using lumping, however, this cannot be obtained. The relationship between the introduced weld volume and its contact surfaces that are available for heat dissipation is now imbalanced. This mismatch results in a more profound annealing effect which again affects the residual stresses and deformations. Reducing energy input corresponding to those heat dissipating contact surfaces did not lead to any significant improvement in the calculation result.

Furthermore, some results appeared to be close to experiments. Although it is important to note that this most likely is by accident and, thus, might be misleading. In most cases numerical engineers will need to validate their models through distortion or X-ray measurements, if any. Though, it could be that apparently correct near surface residual stresses do not necessarily result in true through-thickness residual stress distributions.

\section{References}

[1] M. P. Macherauch E., „Das $\sin ^{2} \psi$-Verfahren der röntgenografischen Spannungsmessung,“ Zeitschrift für angewandte Physik, Bd. 13, 1961.

[2] C. Rohrbach, Handbuch für experimentelle Spannungsanalyse, Düsseldorf: VDI-Verlag GmbH, 1989.

[3] L.-E. Lindgren, H. Runnemalm und M. O. Näsström, „Simulation of multipass welding of a thick plate,“ International Journal for Numerical Methods in Engineering, Nr. 44, pp. 1301-1316, 1999.

[4] L.-E. Lindgren und E. Hedblom, „Modelling of addition of filler material in large deformation analysis of multipass welding," Communications in Numerical Methods in Engineering, Nr. 17, pp. 647-657, 17 August 2001.

[5] D. W. Lobitz, J. D. McClure und R. E. Nickell, „Residual stresses and distortions in multipass welding, “ in Numerical Modeling of Manufacturing Processes, Winter Annual Meeting of the American Society of Mechanical Engineers, Atlanta, Giorgia, 1977.

[6] J. Klassen, „Beitrag zur vereinfachten Eigenspannungsberechnung von Mehrlagenschweißverbindungen,“ Shaker Verlag, Aachen, 2018.

[7] K. Dilger, W. Fricke und T. Nitschke-Pagel, „Entwicklung von Methodiken zur Bewertung von Eigenspannungen an Montagestößen bei Stahl-Großstrukturen,“ AiF-Schlussbericht, IGFVorhabennummer 17652N (in German), 2016

[8] F. Zhang, „Beitrag zum schweißbedingten Verzug unter Berücksichtigung seiner Wechselwirkung mit den Eigenspannungen,“ Institut für Schweißtechnik und Werkstofftechnologie, Braunschweig, 1998. 